\title{
Bregman Distance and Strong Convergence of Proximal-Type Algorithms
}

\author{
Li-Wei Kuo' ${ }^{1}$ and D. R. Sahu ${ }^{2}$ \\ ${ }^{1}$ Department of Applied Mathematics, National Sun Yat-Sen University, Kaohsiung 80424, Taiwan \\ ${ }^{2}$ Department of Mathematics, Banaras Hindu University, Varanasi 221005, India
}

Correspondence should be addressed to Li-Wei Kuo; mpu.verilog@gmail.com

Received 31 October 2012; Revised 7 March 2013; Accepted 7 March 2013

Academic Editor: Dumitru Motreanu

Copyright (c) 2013 L.-W. Kuo and D. R. Sahu. This is an open access article distributed under the Creative Commons Attribution License, which permits unrestricted use, distribution, and reproduction in any medium, provided the original work is properly cited.

The purpose of this paper is to discuss some fundamental properties of Bregman distance, generalized projection operators, firmly nonexpansive mappings, and resolvent operators of set-valued monotone operators corresponding to a functional $\Phi(\|\cdot\|)$. We further study some proximal point algorithms for finding zeros of monotone operators and solving generalized mixed equilibrium problems in Banach spaces. Our results improve and extend some recent results concerning generalized projection operators corresponding to Bregman distance.

\section{Introduction}

In this paper, $X$ denotes a real Banach space with norm $\|\cdot\|$, and $X^{*}$ denotes the Banach dual of $X$ endowed with the dual norm $\|\cdot\|_{*}$. We write $\langle x, j\rangle$ for the value of a functional $j$ in $X^{*}$ at $x$ in $X$. As usual, $x_{\lambda} \rightarrow x$ and $x_{\lambda} \rightarrow x$ stand for the norm and weak convergence of a net $\left\{x_{\lambda}\right\}$ to $x$ in $X$, respectively.

A continuous strictly increasing function $\varphi: \mathbb{R}^{+} \rightarrow \mathbb{R}^{+}$ is said to be a gauge if

$$
\varphi(0)=0, \quad \lim _{t \rightarrow+\infty} \varphi(t)=+\infty
$$

The mapping $J_{\varphi}: X \rightarrow 2^{X^{*}}$ defined by

$$
\begin{aligned}
& J_{\varphi}(x) \\
& =\left\{j \in X^{*}:\langle x, j\rangle=\|x\|\|j\|_{*},\|j\|_{*}=\varphi(\|x\|)\right\}, \quad x \in X,
\end{aligned}
$$

is called the duality mapping with gauge $\varphi$. In the special case where $\varphi(t)=t$, the duality mapping $J_{\varphi}=: J$ is the classical normalized duality mapping. In the case $\varphi(t)=t^{p-1}, p>1$, the duality mapping $J_{\varphi}=: J_{p}$ is called the generalized duality mapping and it is given by

$$
\begin{aligned}
& J_{p}(x) \\
& :=\left\{j \in X^{*}:\langle x, j\rangle=\|x\|\|j\|_{*},\|j\|_{*}=\|x\|^{p-1}\right\}, \quad x \in X .
\end{aligned}
$$

For a gauge $\varphi$, the function $\Phi: \mathbb{R}^{+} \rightarrow \mathbb{R}^{+}$defined by

$$
\Phi(t)=\int_{0}^{t} \varphi(s) d s
$$

is a continuous convex strictly increasing differentiable function on $\mathbb{R}^{+}$with $\Phi^{\prime}(t)=\varphi(t)$ and $\lim _{t \rightarrow+\infty} \Phi(t) / t=+\infty$. Therefore, $\Phi$ has a continuous inverse function $\Phi^{-1}$.

We recall the Bregman Distance $D_{\varphi}$ and function $D_{\varphi}^{f}$ studied in [1]. Let $X$ be a real smooth Banach space. The Bregman distance $D_{\varphi}(x, y)$ between $x$ and $y$ in $X$ is defined by

$$
D_{\varphi}(x, y):=\Phi(\|x\|)-\Phi(\|y\|)-\left\langle x-y, J_{\varphi}(y)\right\rangle .
$$

One can see from Lemma 3 that $D_{\varphi}(x, y) \geq 0$. In the case $\varphi(t)=t^{p-1}, p \in(1, \infty)$, the distance $D_{\varphi}(x, y)=: D_{p}(x, y)$ is 
called the p-Lyapunov functional studied in [2] and it is given by

$$
D_{p}(x, y)=\frac{\|x\|^{p}}{p}+\frac{\|y\|^{p}}{q}-\left\langle x, J_{p}(y)\right\rangle .
$$

Note that

$$
\phi(x, y):=2 D_{2}(x, y)=\|x\|^{2}-2\langle x, J y\rangle+\|y\|^{2}
$$

is the Lyapunov functional. It is obvious that

$$
(\|x\|-\|y\|)^{2} \leq \phi(x, y), \quad \forall x, y \in X .
$$

See Brègman [3], Butnariu and Iusem [4], and Censor and Lent [5].

Let $C$ be a nonempty closed convex subset of a smooth Banach space $X$. The generalized projection $\Pi_{C}: X \rightarrow C$ is defined by

$$
\Pi_{C}(x)=\left\{x_{0} \in C: \phi\left(x_{0}, x\right)=\inf _{y \in C} \phi(y, x)\right\} .
$$

The metric projection operator $P_{C}: X \rightarrow C$ defined by

$$
P_{C} x:=\underset{y \in C}{\arg \min } \frac{1}{2}\|x-y\|^{2}, \quad x \in X,
$$

has been employed successfully in optimization, optimal control, approximation theory, and fixed point theory in the framework of Hilbert spaces. In such a framework, metric projections $P_{C}$ are nonexpansive (i.e., $\left\|P_{C} x-P_{C} y\right\| \leq\|x-y\|$ for all $x, y$ in $H$ ). However, this is no longer true in the framework of Banach spaces. Instead, the generalized projections $\Pi_{C}$ are needed. In [6], Alber generalized the metric projection operator $P_{C}$ to generalized projection operators $\Pi_{C}: X \rightarrow$ $C$ from Hilbert spaces to uniformly smooth Banach spaces. Many applications of the generalized projections in Banach spaces are discussed in the recent literature (see [7-12]).

Section 2 contains preliminaries. In Section 3, we study the fundamental properties of Bregman distance $D_{\varphi}$ and $(\varphi, f)$-generalized projection operators, where $f: X \rightarrow$ $\mathbb{R}^{+}$is a proper, convex, lower semicontinuous function. In Section 4 , we discuss $\varphi$-firmly nonexpansive mappings and $\varphi$-resolvent operators. In Section 5, we establish strong convergence of the proximal-projection methods for finding fixed points of $\varphi$-firmly nonexpansive mappings, zeros of (not necessarily maximal) monotone operators, and solutions of generalized mixed equilibrium problems in Banach spaces using $(\varphi, f)$-generalized projection operators $\Pi_{C}^{\varphi, f}$. Here, we do not assume the maximality of monotone operators and the uniform smoothness of Banach spaces.

\section{Preliminaries}

Let $A: X \rightarrow 2^{X^{*}}$ be a set-valued operator. The set $\mathscr{D}(A)=$ $\{x \in X: A x \neq \emptyset\}$ is called the effective domain of $A$. The range of $A$ is defined by $\mathscr{R}(A)=\cup_{x \in \mathscr{D}(A)} A x$. The operator $A$ is said to be monotone if for any $x, y$ in $\mathscr{D}(A)$, we have

$$
j_{x} \in A x, \quad j_{y} \in A y \Longrightarrow\left\langle x-y, j_{x}-j_{y}\right\rangle \geq 0 .
$$

A monotone operator $A$ is said to be maximal if the graph $\mathscr{G}(A)=\{(x, y): x \in \mathscr{D}(A), y \in A x\}$ of $A$ is not a proper subset of the graph of another monotone operator. We know that if $A$ is a maximal monotone operator, then the zero set $A^{-1} 0$ is closed and convex.

In the rest of this paper, by $\varphi$ we always mean a gauge and by $\Phi$ the corresponding function defined in (4). We list some properties of the duality mapping $J_{\varphi}: X \rightarrow 2^{X^{*}}$ below (for more details see $[13,14])$.

\section{Proposition 1. Let $X$ be a real Banach space.}

(i) $J_{\varphi}$ is norm-to-weak ${ }^{*}$ upper semicontinuous;

(ii) for each $x$ in $X$, the set $J_{\varphi}(x)$ is convex and weakly closed in $X^{*}$;

(iii) $J_{\varphi}(-x)=-J_{\varphi}(x)$ and $J_{\varphi}(\lambda x)=$ $(\varphi(\|\lambda x\|) / \varphi(\|x\|)) J_{\varphi}(x)$ for all nonzero $x$ in $X, \lambda>0$;

(iv) there holds

$$
J_{\varphi}(x)=\frac{\varphi(\|x\|)}{\|x\|} J(x), \quad 0 \neq x \in X
$$

(v) $J_{\varphi}$ is maximal monotone;

(vi) if $X$ is strictly convex, then $J_{\varphi}$ is strictly monotone; that is,

$$
\left\langle x-y, j_{x}-j_{y}\right\rangle>0 \quad \forall x, y \in X, j_{x} \in J_{\varphi}(x), j_{y} \in J_{\varphi}(y) ;
$$

(vii) if $X$ is strictly convex and reflexive, then $J_{\varphi}$ is singlevalued monotone and demicontinuous.

The following result is well known. We include a proof for completeness.

Lemma 2. If a Banach space $E$ has a uniformly Gâteaux differentiable norm, then $J: E \rightarrow E^{*}$ is uniformly norm-toweak ${ }^{*}$ continuous on nonempty bounded subsets of $E$ to $E^{*}$.

Proof. Suppose not, and there exist norm one vectors $x_{n}, y_{n}, z$ in $E$ and a constant $\epsilon>0$ such that $y_{n}-x_{n} \rightarrow 0$, and $\left\langle z, J\left(y_{n}\right)-J\left(x_{n}\right)\right\rangle \geq \epsilon$, for all $n \in \mathbb{N}$. For a fixed $t>0$, define

$$
\begin{aligned}
& a_{n}:=\frac{\left\|x_{n}+t z\right\|-\left\|x_{n}\right\|-t\left\langle z, J\left(x_{n}\right)\right\rangle}{t}, \\
& b_{n}:=\frac{\left\|y_{n}+t z\right\|-\left\|y_{n}\right\|-t\left\langle z, J\left(y_{n}\right)\right\rangle}{t} .
\end{aligned}
$$

Observe that

$$
\begin{aligned}
a_{n} & \geq \frac{\left\langle x_{n}+t z, J\left(y_{n}\right)\right\rangle-\left\langle x_{n}+t z, J\left(x_{n}\right)\right\rangle}{t} \\
& =\left\langle z, J\left(y_{n}\right)-J\left(x_{n}\right)\right\rangle+\frac{\left\langle x_{n}, J\left(y_{n}\right)-J\left(x_{n}\right)\right\rangle}{t}, \\
b_{n} & \geq \frac{\left\langle y_{n}+t z, J\left(x_{n}\right)\right\rangle-\left\langle y_{n}+t z, J\left(y_{n}\right)\right\rangle}{t} \\
& =\left\langle z, J\left(y_{n}\right)-J\left(x_{n}\right)\right\rangle+\frac{\left\langle y_{n}, J\left(y_{n}\right)-J\left(x_{n}\right)\right\rangle}{t} .
\end{aligned}
$$


Hence,

$$
\begin{aligned}
a_{n}+b_{n} \geq & 2\left\langle z, J\left(y_{n}\right)-J\left(x_{n}\right)\right\rangle \\
& -\frac{\left\langle x_{n}-y_{n}, J\left(y_{n}\right)-J\left(x_{n}\right)\right\rangle}{t} \\
\geq & 2 \epsilon-2 \frac{\left\|x_{n}-y_{n}\right\|}{t} .
\end{aligned}
$$

Choose $t=(2 / \epsilon)\left\|x_{n}-y_{n}\right\|$. By the uniform Gâteaux differentiability of the norm, if $n$ is large enough, both $a_{n}$ and $b_{n}$ are less than $(1 / 2) \epsilon$, and so $a_{n}+b_{n}<\epsilon$. We arrive at a contradiction.

Together with Proposition 1, the conclusion in Lemma 2 also holds for $J_{\varphi}$.

Let $X$ be a Banach space and $\psi: X \rightarrow(-\infty,+\infty]$ a function. The function $\psi$ is proper if $\operatorname{dom}(\psi):=\{x \in$ $X: \psi(x)<+\infty\} \neq \emptyset$. An element $j$ in $X^{*}$ is said to be a subgradient of a proper convex function $\psi: X \rightarrow(-\infty,+\infty]$ at a point $x$ in $\operatorname{dom}(\psi)$ if

$$
\psi(x)-\psi(y) \leq\langle x-y, j\rangle, \quad \forall y \in X
$$

The set (possibly empty)

$$
\partial \psi(x):=\left\{j \in X^{*}: \psi(x)-\psi(y) \leq\langle x-y, j\rangle \forall y \in X\right\},
$$

of subgradients of $\psi$ at $x$ in $\operatorname{dom}(\psi)$, is called the subdifferential of $\varphi$ at $x$.

Lemma 3. Let $X$ be a smooth Banach space and $J_{\varphi}: X \rightarrow X^{*}$ the duality mapping with gauge $\varphi$. Then $J_{\varphi}(y)=\partial \Phi(\|y\|)$ for $y \in X \backslash\{0\}$; that is,

$$
\Phi(\|x\|)-\Phi(\|y\|)-\left\langle x-y, J_{\varphi}(y)\right\rangle \geq 0, \quad \forall x \in X .
$$

The proof of the following result is straightforward.

Lemma 4. Let $X$ be a real Banach space and $\varphi$ a gauge function.

(a) $\Phi(\|\cdot\|)$ is a convex and continuous function on $X$.

(b) $X$ is strictly convex if and only if $\Phi(\|\cdot\|)$ is strictly convex.

Lemma 5. Let $X$ be a real Banach space and $\varphi$ a gauge function. Then the following assertions are equivalent:

(a) $X$ is uniformly convex;

(b) $\Phi(\|\cdot\|)$ is uniformly convex on the closed ball $B_{r}:=$ $\{x \in X:\|x\| \leq r\}$, where $r>0$ is arbitrarily given. That is, there exists a strictly increasing convex function $g_{r}: \mathbb{R}^{+} \rightarrow \mathbb{R}^{+}$with $g_{r}(0)=0$ such that

$$
\begin{gathered}
\Phi(\|t x+(1-t) y\|) \leq t \Phi(\|x\|)+(1-t) \Phi(\|y\|) \\
-t(1-t) g_{r}(\|x-y\|)
\end{gathered}
$$

for all $x, y$ in $B_{r}$ and $t$ in $[0,1]$.
Proof. First we note that the general case can be reduced to the case $r=1$. Suppose it holds

$$
\begin{gathered}
\Phi(\|t x+(1-t) y\|) \leq t \Phi(\|x\|)+(1-t) \Phi(\|y\|) \\
-t(1-t) g(\|x-y\|) .
\end{gathered}
$$

We will show that (20) holds for any $r>0$. Set $\varphi_{r}(t)=\varphi(r t)$ and $g_{r}(t)=r g(t / r)$ for $t \geq 0$. Then $\varphi_{r}$ is still a gauge function, and let $\Phi_{r}$ be the function corresponding to $\varphi_{r}$ as defined in (4), $r \Phi_{r}(t)=\Phi(r t)$. Let $x, y \in B_{r}$ and $x_{r}=x / r$ and $y_{r}=y / r$. Then $x_{r}, y_{r} \in B_{X}$. Applying (21) to $\Phi_{r}$, we get

$$
\begin{aligned}
\Phi(\|t x+(1-t) y\|) \\
=r \Phi_{r}\left(\left\|t x_{r}+(1-t) y_{r}\right\|\right) \\
\leq r t \Phi_{r}\left(\left\|x_{r}\right\|\right)+r(1-t) \Phi_{r}\left(\left\|y_{r}\right\|\right) \\
\quad-r t(1-t) g\left(\left\|x_{r}-y_{r}\right\|\right) \\
=t \Phi(\|x\|)+(1-t) \Phi(\|y\|) \\
\quad-t(1-t) g_{r}(\|x-y\|),
\end{aligned}
$$

which is exactly (20). A similar argument also shows that the case $r=1$ can be deduced from any other case $r>0$.

Below, we assume $r=1$ and $g=g_{1}$.

(b) $\Rightarrow$ (a). Given $x, y$ in $X$ such that $\|x\|=\|y\|=1$ and $\|x-y\|=\varepsilon$. Setting $t=1 / 2$ in (20), we have

$$
\Phi\left(\left\|\frac{x+y}{2}\right\|\right) \leq \Phi(1)-\frac{1}{4} g(\varepsilon) .
$$

It turns out that

$$
1-\left\|\frac{x+y}{2}\right\| \geq 1-\Phi^{-1}\left(\Phi(1)-\frac{1}{4} g(\varepsilon)\right)>0 .
$$

This verifies that $X$ is uniformly convex.

(a) $\Rightarrow$ (b). Assume that $X$ is uniformly convex, which implies that $\Phi(\|\cdot\|)$ is strictly convex by Lemma 4(b). Define a function $\mu$ on $[0,2]$ by setting $\mu(0)=0$, and for $0<\varepsilon \leq 2$,

$$
\begin{aligned}
\mu(\varepsilon):=\inf \{ & \frac{1}{2} \Phi(\|x\|)+\frac{1}{2} \Phi(\|y\|) \\
& \left.-\Phi\left(\frac{1}{2}\|x+y\|\right): x, y \in B_{X},\|x-y\| \geq \varepsilon\right\},
\end{aligned}
$$

where $B_{X}$ is the closed unit ball of $X$.

Claim. Consider $\mu(\varepsilon)>0$ for $0<\varepsilon \leq 2$.

Suppose on the contrary that $\mu(\varepsilon)=0$ for some $0<\varepsilon \leq 2$. Then we can find sequences $\left\{x_{n}\right\},\left\{y_{n}\right\}$ in $B_{X}$ such that $\| x_{n}-$ $y_{n} \| \geq \varepsilon$ for all $n$, and

$$
\frac{1}{2} \Phi\left(\left\|x_{n}\right\|\right)+\frac{1}{2} \Phi\left(\left\|y_{n}\right\|\right)-\Phi\left(\frac{\left\|x_{n}+y_{n}\right\|}{2}\right) \longrightarrow 0 .
$$

Without loss of generality, we may assume that

$$
\begin{gathered}
\left\|x_{n}\right\| \longrightarrow \alpha, \quad\left\|y_{n}\right\| \longrightarrow \beta, \\
\left\|x_{n}+y_{n}\right\| \longrightarrow \gamma, \quad\left\|x_{n}-y_{n}\right\| \longrightarrow \theta \geq \varepsilon .
\end{gathered}
$$


It then follows from (26) that

$$
\frac{1}{2} \Phi(\alpha)+\frac{1}{2} \Phi(\beta)=\Phi\left(\frac{\gamma}{2}\right)
$$

The strict convexity of $\Phi$ together with (28) implies that $\alpha+$ $\beta<\gamma$ if $\alpha \neq \beta$. Since, on the other hand, by definition, $\gamma \leq$ $\alpha+\beta$. We therefore must have $\alpha=\beta$, which together with (28) implies that $\alpha=\beta=\gamma / 2$. If we set $x_{n}^{\prime}=x_{n} /\left\|x_{n}\right\|, y_{n}^{\prime}=$ $y_{n} /\left\|y_{n}\right\|$, then $\left\|x_{n}^{\prime}\right\|=\left\|y_{n}^{\prime}\right\|=1$ for all $n$; moreover, from (27), we get

$$
\left\|\frac{x_{n}^{\prime}+y_{n}^{\prime}}{2}\right\| \longrightarrow 1, \quad\left\|x_{n}^{\prime}-y_{n}^{\prime}\right\| \longrightarrow \frac{\theta}{\alpha}>0 .
$$

This contradicts the uniform convexity of $X$, and verifies that $\mu(\varepsilon)>0$ for all $0<\varepsilon \leq 2$.

It turns out from (25) that

$$
\begin{aligned}
\Phi\left(\left\|\frac{1}{2} x+\frac{1}{2} y\right\|\right) \leq & \frac{1}{2} \Phi(\|x\|)+\frac{1}{2} \Phi(\|y\|) \\
& -\frac{1}{4} g(\|x-y\|),
\end{aligned}
$$

for all $x, y$ in $B_{X}$, with $g=4 \mu$.

By the dyadic rational argument used in the proof of $[15$, Theorem 2.2], we can extend the inequality (30) to the case of a general convex combination of $x$ and $y$, namely,

$$
\begin{gathered}
\Phi(\|t x+(1-t) y\|) \leq t \Phi(\|x\|)+(1-t) \Phi(\|y\|) \\
-t(1-t) g(\|x-y\|)
\end{gathered}
$$

for $x, y$ in $B_{X}$ and $t$ in $[0,1]$. Note that $g$ is increasing and continuous. By [16], the function $g$ can also be assumed to be convex (the convexity of $g$ is not needed in our argument throughout the rest of this paper however).

Lemma 6. Let $X$ be a real uniformly convex Banach space. Then there exists a strictly increasing convex function $g$ : $\mathbb{R}^{+} \rightarrow \mathbb{R}^{+}$with $g(0)=0$ such that

$$
\begin{array}{r}
\Phi(\|x+y\|) \geq \Phi(\|x\|)+\langle y, j\rangle+g(\|y\|), \\
\forall x, y \in B_{X}, \forall j \in J_{\varphi}(x) .
\end{array}
$$

Proof. Since $J_{\varphi}$ is the subdifferential of the functional $\Phi(\|\cdot\|)$, we have for $j_{x}$ in $J_{\varphi}(x), x$ in $X$ that

$$
\left\langle y-x, j_{x}\right\rangle \leq \Phi(\|y\|)-\Phi(\|x\|) \quad \forall y \in X
$$

Let $g$ be the function that satisfies (20) with $r=2$ and assume that $x, y \in B_{X}$. Replacing $y$ with $x+\lambda y, 0<\lambda<1$, we obtain

$$
\begin{aligned}
\left\langle y, j_{x}\right\rangle \leq & \frac{[\Phi(\|x+\lambda y\|)-\Phi(\|x\|)]}{\lambda} \\
= & \frac{[\Phi(\|(1-\lambda) x+\lambda(x+y)\|)-\Phi(\|x\|)]}{\lambda} \\
\leq & {[(1-\lambda) \Phi(\|x\|)+\lambda \Phi(\|x+y\|)} \\
& \quad-\lambda(1-\lambda) g(\|y\|)-\Phi(\|x\|)](\lambda)^{-1} \\
\leq & \Phi(\|x+y\|)-\Phi(\|x\|)-(1-\lambda) g(\|y\|) .
\end{aligned}
$$

Taking limit as $\lambda \rightarrow 0$, we get

$$
\left\langle y, j_{x}\right\rangle=\Phi(\|x+y\|)-\Phi(\|x\|)-g(\|y\|) .
$$

Lemma 7 (see [17, Theorem 3.11, page 952]). Let $X$ be a reflexive Banach space and $J_{\varphi}: X \rightarrow 2^{X^{*}}$ the duality map with gauge $\varphi$. Suppose that $A: X \rightarrow 2^{X^{*}}$ is maximal monotone. Then the operator $A+J_{\varphi}$ is surjective.

\section{Bregman Distance $D_{\varphi}$ and Function $D_{\varphi}^{f}$}

One can easily see that

$$
\begin{aligned}
& D_{\varphi}(x, y)+D_{\varphi}(y, z)-D_{\varphi}(x, z) \\
& \quad=\left\langle x-y, J_{\varphi}(z)-J_{\varphi}(y)\right\rangle, \quad \forall x, y, z \in X .
\end{aligned}
$$

Noticing that for $x$ in $X$, the scalar function $D_{\varphi}(\cdot, x)$ is coercive (see [18, Lemma $7.3(\mathrm{v})]$ ).

Proposition 8. Let $X$ be a strictly convex smooth Banach space. Let $x, y \in X$. Then

$$
D_{\varphi}(x, y)=0 \Longleftrightarrow x=y .
$$

Proof. See [18, Lemma 7.3(vi)]

Proposition 9. Let $X$ be a smooth and uniformly convex Banach space. Then there exists a strictly increasing convex function $g: \mathbb{R}^{+} \rightarrow \mathbb{R}^{+}$with $g(0)=0$ such that

$$
D_{\varphi}(u, v) \geq g(\|u-v\|) \quad \forall u, v \in B_{X} .
$$

Proof. Let $u, v \in B_{X}$. By Lemma 6 we have

$$
\begin{aligned}
D_{\varphi}(u, v)= & \Phi(\|v+(u-v)\|)-\Phi(\|v\|) \\
& -\left\langle u-v, J_{\varphi}(v)\right\rangle \\
\geq & \Phi(\|v\|)+\left\langle u-v, J_{\varphi}(v)\right\rangle+g(\|u-v\|) \\
& -\Phi(\|v\|)-\left\langle u-v, J_{\varphi}(v)\right\rangle \\
= & g(\|u-v\|) .
\end{aligned}
$$

As in Butnariu et al. [19], we can prove the following proposition.

Proposition 10. Let $X$ be a smooth and uniformly convex Banach space. Let $\left\{x_{n}\right\}$ and $\left\{y_{n}\right\}$ be two sequences in $X$ such that $D_{\varphi}\left(x_{n}, y_{n}\right) \rightarrow 0$. If $\left\{y_{n}\right\}$ is bounded, then $\left\|x_{n}-y_{n}\right\| \rightarrow 0$.

Proof. Assume $\left\{y_{n}\right\}$ is bounded. From definition (5) we have

$$
\begin{aligned}
D_{\varphi}\left(x_{n}, y_{n}\right)= & \Phi\left(\left\|x_{n}\right\|\right)-\Phi\left(\left\|y_{n}\right\|\right) \\
& -\left\langle x_{n}-y_{n}, J_{\varphi}\left(y_{n}\right)\right\rangle .
\end{aligned}
$$


It follows that

$$
\begin{aligned}
& \Phi\left(\left\|x_{n}\right\|\right)-\left\|x_{n}\right\| \varphi\left(\left\|y_{n}\right\|\right) \\
& \leq \Phi\left(\left\|x_{n}\right\|\right)-\left\langle x_{n}, J_{\varphi}\left(y_{n}\right)\right\rangle \\
& \quad=\Phi\left(\left\|y_{n}\right\|\right)-\left\|x_{n}\right\| \varphi\left(\left\|y_{n}\right\|\right)+D_{\varphi}\left(x_{n}, y_{n}\right) \\
& \quad \leq \Phi\left(\left\|y_{n}\right\|\right)+D_{\varphi}\left(x_{n}, y_{n}\right) .
\end{aligned}
$$

Since $\left\{y_{n}\right\}$ is bounded, $D_{\varphi}\left(x_{n}, y_{n}\right) \rightarrow 0$ and $\Phi(t) / t \rightarrow \infty$ as $t \rightarrow \infty$ it follows from (41) that $\left\{\left\|x_{n}\right\|\right\}$ is bounded, too.

We may now assume that $\left\{x_{n}\right\}$ and $\left\{y_{n}\right\}$ both lie in the closed unit ball $B_{X}$ (otherwise consider the rescaled sequences $\left\{\gamma x_{n}\right\}$ and $\left\{\gamma y_{n}\right\}$ for a sufficiently small $\gamma>0$ ). By Proposition 9, there exists a strictly increasing convex function $g: \mathbb{R}^{+} \rightarrow \mathbb{R}^{+}$with $g(0)=0$ such that

$$
g\left(\left\|x_{n}\right\|-\left\|y_{n}\right\|\right) \leq D_{\varphi}\left(x_{n}, y_{n}\right), \quad \forall n \in \mathbb{N} .
$$

Since $D_{\varphi}\left(x_{n}, y_{n}\right) \rightarrow 0$ and $g$ is strictly increasing, we immediately conclude that $\left\|x_{n}-y_{n}\right\| \rightarrow 0$.

Proposition 11 (see [20, Lemma 3.1]). Let $X$ be a smooth Banach space. Let $u \in X$ and $\left\{x_{n}\right\}$ be a sequence in $X$ such that $\left\{D_{\varphi}\left(x_{n}, u\right)\right\}$ is bounded. Then $\left\{x_{n}\right\}$ is bounded.

The statement in the following proposition is evident from the definition of $D_{\varphi}$ (cf. [18, Lemma 7.3(ii)]).

Proposition 12. Let $X$ be a smooth Banach space. Then, for any fixed $x$ in $X$, the scalar function $D_{\varphi}(\cdot, x)$ is continuous, weakly lower semicontinuous, and convex on $X$.

Let $f: X \rightarrow[0,+\infty]$ be a proper, convex, lower semicontinuous function. Define

$$
D_{\varphi}^{f}(x, y)=D_{\varphi}(x, y)+f(x), \quad \forall x, y \in X .
$$

Some of the following basic properties of the Bregman distance $D_{\varphi}$ and function $D_{\varphi}^{f}$ are known in the literature (see [18-21]).

The following proposition can be deduced from Butnariu and Kassay [21, Lemma 2.1].

Proposition 13. Let $C$ be a nonempty closed convex subset of a reflexive, strictly convex, and smooth Banach space $X$. Let $x \in X$ and let $f: X \rightarrow[0,+\infty]$ be a proper, convex, lower semicontinuous function with $C \subset \operatorname{dom}(f)$. Then there exists a unique element $x_{0}$ in $C$ such that

$$
D_{\varphi}^{f}\left(x_{0}, x\right)=\inf \left\{D_{\varphi}^{f}(z, x): z \in C\right\} .
$$

Bregman projections are thoroughly studied and used for iteration schemes such as sequential subspace methods or split feasibility problems successfully (see, [22-24]). The notion of $D_{f}$-proximal mappings was introduced and studied in [1]. Recently, the notion of Moreau proximal mapping [25] is generalized by Butnariu and Kassay [21] as the proximal mapping relative to $f$ associated with a proper, convex, lower semicontinuous function $\varphi$. Using the idea of $[1,26]$, Proposition 13 allows us to extend generalized projections $\Pi_{C}$ as follows.
Definition 14. In the setting of Proposition 13, we define the $(\varphi, f)$-generalized projection from $X$ onto $C$ by

$$
\Pi_{C}^{\varphi, f}(x)=x_{0}, \quad x \in X .
$$

In case $\varphi(t)=t$ and $f=0$, we notice that $\Pi_{C}^{\varphi, f}$ coincides with $\Pi_{C}$. In case that $X$ is a Hilbert space and $\varphi(t)=t$, denote $\Pi_{C}^{\varphi, f}$ by $P_{C}^{f}$.

Applying the tools used in $[1,26,27]$, we can establish the following results.

Proposition 15. Let $C$ be a nonempty closed convex subset of a reflexive, strictly convex, and smooth Banach space $X$ and let $f: X \rightarrow[0,+\infty]$ be a proper, convex, lower semicontinuous function with $C \subset \operatorname{dom}(f)$.

(i) Let $x_{0} \in C$ and $x \in X$. Then the following assertions are equivalent:

$$
\begin{aligned}
& \text { (a) } x_{0}=\Pi_{C}^{\varphi, f}(x), \\
& \text { (b) }\left\langle z-x_{0}, J_{\varphi} x_{0}-J_{\varphi} x\right\rangle+f(z)-f\left(x_{0}\right) \geq 0 \text {, } \\
& \forall z \in C \text {. }
\end{aligned}
$$

(ii) Given $x$ in $X$, one has

$$
\begin{aligned}
& D_{\varphi}\left(y, \Pi_{C}^{\varphi, f}(x)\right)+D_{\varphi}\left(\Pi_{C}^{\varphi, f}(x), x\right) \\
& \quad \leq D_{\varphi}(y, x)+f(y)-f\left(\Pi_{C}^{\varphi, f}(x)\right), \quad \forall y \in C .
\end{aligned}
$$

Proposition 16. Let $C$ be a nonempty closed convex subset of a smooth and uniformly convex Banach space $X$ and $f: X \rightarrow$ $[0,+\infty]$ a proper, convex, lower semicontinuous function with $C \subset \operatorname{dom}(f)$. Let $u \in X$ and let $\left\{x_{n}\right\}$ be a bounded sequence in $C$ such that $\lim _{n \rightarrow+\infty} f\left(x_{n}\right)=0$. If $D_{\varphi}^{f}\left(x_{n}, u\right) \leq$ $D_{\varphi}^{f}\left(\Pi_{C}^{\varphi, f}(u), u\right)$ for all $n$ in $\mathbb{N}$, then $\lim _{n \rightarrow+\infty} x_{n}=\Pi_{C}^{\varphi, f}(u)$.

Proof. Set $\Pi_{C}^{\varphi, f}(u)=: \quad \tilde{u}$. Then, by assumption, we have $D_{\varphi}^{f}\left(x_{n}, u\right) \leq D_{\varphi}^{f}(\tilde{u}, u)$ for all $n$ in $\mathbb{N}$. By the three-point identity (36), we have

$$
\begin{aligned}
D_{\varphi}^{f}\left(x_{n}, \tilde{u}\right)= & D_{\varphi}^{f}\left(x_{n}, u\right)+D_{\varphi}^{f}(u, \widetilde{u}) \\
& -\left\langle x_{n}-u, J_{\varphi}(\widetilde{u})-J_{\varphi}(u)\right\rangle-f(u) \\
\leq & D_{\varphi}^{f}(\widetilde{u}, u)+D_{\varphi}^{f}(u, \widetilde{u}) \\
& -\left\langle x_{n}-u, J_{\varphi}(\widetilde{u})-J_{\varphi}(u)\right\rangle-f(u) \\
\leq & \left\langle\widetilde{u}-u, J_{\varphi}(\widetilde{u})-J_{\varphi}(u)\right\rangle \\
& -\left\langle x_{n}-u, J_{\varphi}(\widetilde{u})-J_{\varphi}(u)\right\rangle+f(\widetilde{u}) \\
= & \left\langle\widetilde{u}-x_{n}, J_{\varphi}(\widetilde{u})-J_{\varphi}(u)\right\rangle+f(\widetilde{u}) .
\end{aligned}
$$

Since $\left\{x_{n}\right\}$ is a bounded sequence in $C$, there exists a subsequence $\left\{x_{n_{i}}\right\}$ of $\left\{x_{n}\right\}$ such that $\left\{x_{n_{i}}\right\}$ converges weakly to some element $z$ in $C$. Note that

$$
0 \leq f(z) \leq \liminf _{i \rightarrow+\infty} f\left(x_{n_{i}}\right)=0
$$


that is, $f(z)=0$. Using Proposition 15, we have

$$
\begin{aligned}
& \lim _{i \rightarrow+\infty} \sup _{\varphi} D_{\varphi}^{f}\left(x_{n_{i}}, \widetilde{u}\right) \\
& \quad \leq \lim _{i \rightarrow+\infty} \sup _{i \rightarrow+\infty}\left[\left\langle\widetilde{u}-x_{n_{i}}, J_{\varphi}(\widetilde{u})-J_{\varphi}(u)\right\rangle+f(\widetilde{u})\right] \\
& \quad \leq\left\langle\widetilde{u}-z, J_{\varphi}(\widetilde{u})-J_{\varphi}(u)\right\rangle+f(\widetilde{u}) \\
& \quad \leq f(z),
\end{aligned}
$$

which implies that $\lim _{i \rightarrow+\infty} D_{\varphi}\left(x_{n_{i}}, \widetilde{u}\right)=0$. It follows from Proposition 10 that $\lim _{i \rightarrow \infty} x_{n_{i}}=\tilde{u}$. This implies that $x_{n}$ converges strongly to $\widetilde{u}=\Pi_{C}^{\varphi, f}(u)$.

\section{4. $\varphi$-Firmly Nonexpansive and $\varphi$-Resolvent Operators}

Following [1], we study properties of $\varphi$-firmly nonexpansive mappings in Banach spaces.

Definition 17. Let $X$ be a smooth Banach space, $J_{\varphi}: X \rightarrow X^{*}$ a duality mapping with gauge function $\varphi$, and $C$ a nonempty subset of $X$. An operator $T: C \rightarrow X$ is called $\varphi$-firmly nonexpansive if

$$
\begin{aligned}
& \left\langle T x-T y, J_{\varphi}(T x)-J_{\varphi}(T y)\right\rangle \\
& \quad \leq\left\langle T x-T y, J_{\varphi}(x)-J_{\varphi}(y)\right\rangle, \quad \forall x, y \in C .
\end{aligned}
$$

In the case of $\varphi(t)=t$, inequality (50) reduces to

$$
\begin{array}{r}
\langle T x-T y, J(T x)-J(T y)\rangle \leq\langle T x-T y, J(x)-J(y)\rangle \\
\forall x, y \in C .
\end{array}
$$

If $T$ satisfies condition (51), we call $T$ of firmly nonexpansive type. The class of firmly nonexpansive type operators is studied by Kohsaka and Takahashi [28]. When $X$ is a Hilbert space, inequality (50) reduces to the following inequality about firmly nonexpansive operators in the classical sense (see Goebel and Kirk [29]):

$$
\|T x-T y\|^{2} \leq\langle T x-T y, x-y\rangle, \quad \forall x, y \in C .
$$

We now give useful characterizations of $\varphi$-firmly nonexpansive mappings which can be deduced from the Bregman distance (5).

Proposition 18. Let $X$ be a smooth Banach space and $C a$ nonempty closed convex subset of $X$. Let $T: C \rightarrow C$ be a $\varphi$-firmly nonexpansive mapping. Then

$$
\begin{gathered}
D_{\varphi}(T x, T y)+D_{\varphi}(T y, T x)+D_{\varphi}(T x, x)+D_{\varphi}(T y, y) \\
\leq D_{\varphi}(T x, y)+D_{\varphi}(T y, x) \quad \forall x, y \in C .
\end{gathered}
$$

The geometry of the fixed point set of $\varphi$-firmly nonexpansive mappings is established in Reich and Sabach [30, Lemma 15.5] as follows.
Proposition 19. Let $X$ be a strictly convex smooth Banach space and $C$ a nonempty closed convex subset of $X$. Let $T$ : $C \rightarrow C$ be a $\varphi$-firmly nonexpansive mapping. Then the set $F(T)$ of fixed points of $T$ is closed and convex.

Let $C$ be a nonempty closed convex subset of a smooth Banach space $X$ and let $T: C \rightarrow C$ be a mapping. A point $u$ in $C$ is an asymptotic fixed point of $T$ if $C$ contains a sequence $\left\{x_{n}\right\}$ such that $x_{n} \rightarrow u$ and $\left\|x_{n}-T x_{n}\right\| \rightarrow 0$; see [31]. We denote the set of asymptotic fixed points of $T$ by $\widehat{F}(T)$. A mapping $T: C \rightarrow C$ is relatively $\varphi$-nonexpansive if the following conditions are satisfied:

(i) $F(T)$ is nonempty;

(ii) $D_{\varphi}(p, T x) \leq D_{\varphi}(p, x)$ for all $x$ in $C$ and $p$ in $F(T)$;

(iii) $\widehat{F}(T)=F(T)$.

The class of relatively $\varphi$-nonexpansive mappings is larger than the class of relatively nonexpansive mappings (see [32]).

A mapping $T: C \rightarrow C$ is $\varphi$-firmly quasinonexpansive if $F(T) \neq \emptyset$ and

$$
\left\langle T x-p, J_{\varphi}(x)-J_{\varphi}(T x)\right\rangle \geq 0
$$

for all $x$ in $C$ and $p$ in $F(T)$. From the definition of the Bregman distance (5) and (54), the following proposition follows immediately.

Proposition 20. Let $X$ be a smooth Banach space, $C$ a nonempty subset of $X$, and $T: C \rightarrow C$ a $\varphi$-firmly quasinonexpansive mapping. Then

$$
D_{\varphi}(p, T x)+D_{\varphi}(T x, x) \leq D_{\varphi}(p, x)
$$

for all $x$ in $C$ and $p$ in $F(T)$. 15.6].

The following supplements Reich and Sabach [30, Lemma

Proposition 21. Let $X$ be a strictly convex Banach space with a uniformly Gâteaux differentiable norm. Let $C$ be a nonempty closed convex subset of $X$ and let $T: C \rightarrow C$ be a $\varphi$-firmly nonexpansive mapping. Then $\widehat{F}(T)=F(T)$.

Proof. It is easy to see that $F(T) \subset \widehat{F}(T)$. It remains to prove that $\widehat{F}(T) \subset F(T)$. For this, suppose that $u \in \widehat{F}(T)$. Then, there exists a sequence $\left\{x_{n}\right\}$ in $C$ such that $x_{n} \rightarrow u$ and $\| x_{n}-$ $T x_{n} \| \rightarrow 0$. We need to prove that $u \in F(T)$. Using (53), we get

$$
\begin{aligned}
D_{\varphi}(T u, u) \leq & D_{\varphi}\left(T x_{n}, u\right)+D_{\varphi}\left(T u, x_{n}\right)-D_{\varphi}\left(T x_{n}, T u\right) \\
& -D_{\varphi}\left(T u, T x_{n}\right)-D_{\varphi}\left(T x_{n}, x_{n}\right) .
\end{aligned}
$$

It is not hard to find that (56) is reduced to the relation

$$
\begin{aligned}
D_{\varphi}(T u, u) \leq & \Phi(\|T u\|)-\Phi(\|u\|)+\left\langle u-T x_{n}, J_{\varphi}(u)\right\rangle \\
& +\left\langle T x_{n}-T u, J_{\varphi}\left(x_{n}\right)-J_{\varphi}\left(T x_{n}\right)\right\rangle \\
& +\left\langle T x_{n}-T u, J_{\varphi}(T u)\right\rangle .
\end{aligned}
$$


We note that $T x_{n} \rightarrow u$. Indeed, for any norm one linear functional $\xi$ of $X$, we have

$$
\left\langle T x_{n}-u, \xi\right\rangle=\left\langle T x_{n}-x_{n}, \xi\right\rangle+\left\langle x_{n}-u, \xi\right\rangle .
$$

Both terms in the right-hand side approach zero. Consequently,

$$
\left\langle T x_{n}-T u, J_{\varphi}(T u)\right\rangle \longrightarrow\left\langle u-T u, J_{\varphi}(T u)\right\rangle .
$$

Claim. Consider $\left\langle T x_{n}-T u, J_{\varphi}\left(x_{n}\right)-J_{\varphi}\left(T x_{n}\right)\right\rangle \rightarrow 0$.

Since $x_{n} \rightarrow u$ and $\left\|x_{n}-T x_{n}\right\| \rightarrow 0$, there is a constant $M>0$ such that all $\left\|x_{n}\right\|,\left\|T x_{n}\right\|<M$. It follows from the uniform norm to weak ${ }^{*}$ continuity of $J_{\varphi}$ on bounded subsets (Lemma 2); we have

$$
\left\langle T u, J_{\varphi}\left(x_{n}\right)-J_{\varphi}\left(T x_{n}\right)\right\rangle \longrightarrow 0 .
$$

Observe that

$$
\epsilon_{n}:=\left\langle T x_{n}-x_{n}, J_{\varphi}\left(x_{n}\right)\right\rangle \longrightarrow 0 .
$$

Moreover, $\left|\left\|x_{n}\right\|-\left\|T x_{n}\right\|\right| \leq\left\|x_{n}-T x_{n}\right\| \rightarrow 0$. By the uniform continuity of $\varphi$ on $[0, M]$, we have

$$
\begin{aligned}
\left\langle T x_{n}, J_{\varphi}\left(x_{n}\right)-J_{\varphi}\left(T x_{n}\right)\right\rangle \\
=\left\langle T x_{n}, J_{\varphi}\left(x_{n}\right)\right\rangle-\left\langle T x_{n}, J_{\varphi}\left(T x_{n}\right)\right\rangle \\
=\left\|x_{n}\right\| \varphi\left(\left\|x_{n}\right\|\right)+\epsilon_{n}-\left\|T x_{n}\right\| \varphi\left(\left\|T x_{n}\right\|\right) \\
=\left(\left\|x_{n}\right\|-\left\|T x_{n}\right\|\right) \varphi\left(\left\|x_{n}\right\|\right) \\
\quad+\left\|T x_{n}\right\|\left(\varphi\left(\left\|x_{n}\right\|\right)-\varphi\left(\left\|T x_{n}\right\|\right)\right)+\epsilon_{n} \longrightarrow 0 .
\end{aligned}
$$

The claim is thus verified.

We obtain from (57) and the claim that

$$
D_{\varphi}(T u, u) \leq \Phi(\|T u\|)-\Phi(\|u\|)+\left\langle u-T u, J_{\varphi}(T u)\right\rangle .
$$

This is equivalent to

$$
\left\langle T u-u, J_{\varphi}(T u)-J_{\varphi}(u)\right\rangle \leq 0 .
$$

The strict monotonicity of the duality mapping $J_{\varphi}$ implies that equality must hold. Namely, $T u=u$ or $u \in F(T)$.

The resolvent of an operator $A: X \rightarrow 2^{X^{*}}$ relative to a Gâteaux differentiable function $f$ is introduced and studied in [1]. We define $\varphi$-resolvent operators following $[1,18]$.

Definition 22. Let $C$ be a nonempty closed convex subset of a smooth Banach space $X$ and let $J_{\varphi}: X \rightarrow X^{*}$ be the duality mapping with gauge $\varphi$. Suppose that $A: X \rightarrow 2^{X^{*}}$ is an operator satisfying the range condition

$$
D(A) \subset C \subset J_{\varphi}^{-1} \mathscr{R}\left(J_{\varphi}+r A\right) \quad \forall r>0 .
$$

For each $\lambda>0$, the $\varphi$-resolvent associated with operator $A$ is the operator $R_{\lambda}^{\varphi, A}: C \rightarrow 2^{X}$ defined by

$$
R_{\lambda}^{\varphi, A} x=\left\{z \in X: J_{\varphi}(x) \in\left(J_{\varphi}+\lambda A\right) z\right\}, \quad x \in C .
$$

For $x$ in $C$ and $\lambda$ in $(0, \infty)$, we have

$$
\begin{aligned}
0 \in A x & \Longleftrightarrow J_{\varphi}(x) \in\left(J_{\varphi}+\lambda A\right) x \\
& \Longleftrightarrow x \in\left(J_{\varphi}+\lambda A\right)^{-1} J_{\varphi} x \\
& \Longleftrightarrow x \in F\left(R_{\lambda}^{\varphi, A}\right) .
\end{aligned}
$$

If $A$ is maximal monotone, then, by Lemma 7, we see that condition (65) holds for $C=\overline{D(A)}$.

Remark 23. For smooth $X$ and $\varphi(t)=t^{p-1}$ with $p \in(1,+\infty)$, we have $J_{\varphi}=J_{p}$ and $R^{p, A}=\left(J_{p}+A\right)^{-1} J_{p}$. For $p=2, R^{A}:=$ $R^{2, A}=(J+A)^{-1} \circ J$ and this kind of resolvent operators is studied in the literature (see $[28,33]$ ).

Let $C$ be a nonempty closed convex subset of a reflexive, strictly convex, and smooth Banach space $X$. Let $A: X \rightarrow$ $2^{X^{*}}$ be a monotone operator satisfying the condition $D(A) \subset$ $C \subset J_{\varphi}^{-1} \mathscr{R}\left(J_{\varphi}+\lambda A\right)$, where $\lambda>0$. Using the smoothness and strict convexity of $X$, we obtain that $R_{\lambda}^{\varphi, A}$ is single-valued. The conditions $D(A) \subset C \subset J_{\varphi}^{-1} \mathscr{R}\left(J_{\varphi}+\lambda A\right)$ ensure that $R_{\lambda}^{\varphi, A}$ is the single-valued $\varphi$-resolvent operator form $C$ into $D(A)$. In other words,

$$
R_{\lambda}^{\varphi, A} x=\left(J_{\varphi}+\lambda A\right)^{-1} J_{\varphi} x, \quad \forall x \in C .
$$

Following $[18,28]$, we have the following proposition.

Proposition 24. Let $C$ be a nonempty closed convex subset of a reflexive, strictly convex and smooth Banach space $X$ and let $J_{\varphi}: X \rightarrow X^{*}$ be the duality mapping with gauge $\varphi$. Let $A$ : $X \rightarrow 2^{X^{*}}$ be a monotone operator satisfying the condition $D(A) \subset C \subset J_{\varphi}^{-1} \mathscr{R}\left(J_{\varphi}+\lambda A\right)$, where $\lambda$ is a positive real number. Let $R_{\lambda}^{\varphi, A}$ be a resolvent of $A$, where
(a) $R_{\lambda}^{\varphi, A}$ is $\varphi$-firmly nonexpansive mapping from $C$ into $C$,
(b) $F\left(R_{\lambda}^{\varphi, A}\right)=A^{-1} 0$.

\section{Convergence Theorems}

Let $C$ be a nonempty subset of a Banach space $X$ and $\mathscr{T}=$ $\{T(t): t>0\}$ a family of mappings from $C$ into $C$ with $\bigcap_{t>0} F(T(t)) \neq \emptyset$. Let $\mathscr{G}=\left\{G_{t}: t>0\right\}$ be a family of mappings from $C$ into $C$ such that $\bigcap_{t>0} F(T(t)) \subseteq \bigcap_{t>0} F\left(G_{t}\right)$. We say the family $\mathscr{T}:=\{T(t): t>0\}$ has property $(\mathscr{A})$ with respect to the family $\mathscr{G}=\left\{G_{t}: t>0\right\}$ if the following assertion holds:

for each bounded net $\left\{x_{s}\right\}_{s>0}$ in $C$ if $x_{s}-G_{s} x_{s} \longrightarrow 0$

$$
\text { as } s \longrightarrow+\infty
$$

then $x_{s}-T(t) x_{s} \longrightarrow 0$ as $s \longrightarrow+\infty, \forall t>0$. 
If $\mathscr{T}=\mathscr{G}$ and the above condition holds, then we say $\mathscr{T}:=\{T(s): s>0\}$ has property $(\mathscr{A})$.

Remark 25. If $\mathscr{T}$ is a singleton, that is, $\mathscr{T}=\{T\}$, or $T(s)=T$ for all $s>0$, then $\{T\}$ always has property $(\mathscr{A})$.

We now give some examples.

Example 26. Let $C$ be a nonempty closed convex subset of a Banach space $X$ and $T$ a nonexpansive mapping from $C$ into $C$ with $F(T) \neq \emptyset$. Assume $b_{t}$ in $\mathbb{R}$ with $0<a \leq b_{t} \leq b<1$ for all $t>0$. Define $G_{t}: C \rightarrow C$ by $G_{t} x=\left(1-b_{t}\right) x+b_{t} T x$ for all $x$ in $C$. Then $T$ has property $(\mathscr{A})$ with respect to the family $\left\{G_{t}: t>0\right\}$.

Proof. Let $\left\{x_{t}\right\}_{t>0}$ be a bounded net in $C$ such that $\| x_{t}-$ $G_{t}\left(x_{t}\right) \| \rightarrow 0$ as $t \rightarrow \infty$. Note that

$$
\left\|x_{t}-T x_{t}\right\|=\frac{1}{b_{t}}\left\|x_{t}-G_{t}\left(x_{t}\right)\right\|
$$

and $0<a \leq b_{t} \leq b<1$ for all $t>0$. Therefore, $\left\|x_{t}-T x_{t}\right\| \rightarrow$ 0 as $t \rightarrow+\infty$.

The following example shows that the family $\left\{R_{t}^{A}: t>\right.$ 0 \} of resolvent operators of a maximal monotone operator $A$ enjoys property $(\mathscr{A})$.

Example 27. Let $C$ be a nonempty closed convex subset of a real Hilbert space $H$ and let $A \subset H \times H$ be a monotone operator satisfying the following condition:

$$
D(A) \subset C \subset \bigcap_{r>0} \mathscr{R}(I+r A) .
$$

Let $\left\{z_{t}\right\}_{t>0}$ be a bounded net in $C$ such that $\left\|z_{t}-R_{t}^{A} z_{t}\right\| \rightarrow 0$ as $t \rightarrow+\infty$. Then $\left\|z_{t}-R_{r}^{A} z_{t}\right\| \rightarrow 0$ as $t \rightarrow+\infty$ for each $r>0$.

Proof. Let $r, t>0$. By Takahashi [34], we have

$$
\frac{1}{r}\left\|R_{t}^{A} z_{t}-R_{r}^{A} R_{t}^{A} z_{t}\right\| \leq \frac{1}{t}\left\|z_{t}-R_{t}^{A} z_{t}\right\| .
$$

Using (72), we have

$$
\begin{aligned}
\left\|z_{t}-R_{r}^{A} z_{t}\right\| \leq & \left\|z_{t}-R_{t}^{A} z_{t}\right\|+\left\|R_{t}^{A} z_{t}-R_{r}^{A} R_{t}^{A} z_{t}\right\| \\
& +\left\|R_{r}^{A} R_{t}^{A} z_{t}-R_{r}^{A} z_{t}\right\| \\
\leq & \left(2+\frac{r}{t}\right)\left\|z_{t}-R_{t}^{A} z_{t}\right\| \longrightarrow 0 \quad \text { as } t \longrightarrow+\infty .
\end{aligned}
$$

We now discuss the problem of finding common fixed points of a sequence of $\varphi$-firmly nonexpansive mappings. Our proximal-projection method is based on (a not necessarily Bregman distance) function $D_{\varphi}^{f}$. The proof is based on the technique in [20].
Theorem 28. Let $X$ be a uniformly convex Banach space with a uniformly Gâteaux differentiable norm. Let $\varphi$ be a gauge function, $C$ a nonempty closed convex subset of $X$, and $f: X \rightarrow[0,+\infty]$ a proper, convex, lower semicontinuous function with $C \subset \operatorname{dom}(f)$. Let $T: C \rightarrow C$ be a $\varphi$ firmly nonexpansive mapping and $\mathscr{T}:=\left\{T_{n}\right\}$ a sequence of $\varphi$-firmly nonexpansive self-mappings on $C$ such that $F(T)=$ $\bigcap_{n \in \mathbb{N}} F\left(T_{n}\right) \neq \emptyset$ and $\mathscr{T}$ has property $(\mathscr{A})$ with respect to $T$. For $u$ in $C$ and $C_{1}=C$ with $x_{1}=\Pi_{C_{1}}^{\varphi, f}(u)$, define a sequence $\left\{x_{n}\right\}$ in $C$ as follows:

$$
\begin{gathered}
y_{n}=T_{n} x_{n}, \\
C_{n+1}=\left\{z \in C_{n}:\left\langle z-y_{n}, J_{\varphi}\left(x_{n}\right)-J_{\varphi}\left(y_{n}\right)\right\rangle \leq 0\right\}, \\
x_{n+1}=\prod_{C_{n+1}}^{\varphi, f}(u) \quad \forall n \in \mathbb{N} .
\end{gathered}
$$

Then $\left\{x_{n}\right\}$ converges strongly to $\Pi_{F(T)}^{\varphi, f}(u)$.

Proof. We proceed the proof in the following steps:

Step 1. $\left\{x_{n}\right\}$ is well defined.

Note that all $C_{n}$ are closed and convex. For $p$ in $F(\mathscr{T})$ and $n$ in $\mathbb{N}$, we obtain from (54) that

$$
\left\langle p-y_{n}, J_{\varphi}\left(x_{n}\right)-J_{\varphi}\left(y_{n}\right)\right\rangle \leq 0
$$

It follows that $p \in C_{n}$ and hence $F(\mathscr{T}) \subset C_{n}$. Therefore, $\left\{x_{n}\right\}$ is well defined.

Step 2. $\left\{x_{n}\right\}$ is bounded.

Let $p \in F(\mathscr{T})$. It follows from Proposition 15; we have

$$
\begin{aligned}
D_{\varphi}\left(x_{n}, u\right)= & D_{\varphi}\left(\Pi_{C_{n}}^{\varphi, f}(u), u\right) \\
\leq & D_{\varphi}(p, u)-D_{\varphi}\left(p, \Pi_{C_{n}}^{\varphi, f}(u)\right) \\
& \quad+f(p)-f\left(x_{n}\right) \\
\leq & D_{\varphi}(p, u)+f(p) .
\end{aligned}
$$

It follows that $\left\{D_{\varphi}\left(x_{n}, u\right)\right\}$ is bounded and hence from Proposition 11, we obtain that $\left\{x_{n}\right\}$ is bounded.

Step 3. Consider $\left\|x_{n}-T x_{n}\right\| \rightarrow 0$.

Note that $x_{n+1} \in C_{n+1} \subset C_{n}$. It follows from Proposition 15 that

$$
\begin{aligned}
& D_{\varphi}\left(x_{n+1}, \Pi_{C_{n}}^{\varphi, f}(u)\right)+D_{\varphi}\left(\Pi_{C_{n}}^{\varphi, f}(u), u\right) \\
& \quad \leq D_{\varphi}\left(x_{n+1}, u\right)+f\left(x_{n+1}\right)-f\left(\Pi_{C_{n}}^{\varphi, f}(u)\right) .
\end{aligned}
$$

This implies that

$$
\begin{aligned}
D_{\varphi}\left(x_{n}, u\right) \leq & D_{\varphi}\left(x_{n+1}, u\right)-D_{\varphi}\left(x_{n+1}, x_{n}\right) \\
& +f\left(x_{n+1}\right)-f\left(x_{n}\right) .
\end{aligned}
$$


Therefore, the sequence $\left\{D_{\varphi}^{f}\left(x_{n}, u\right)\right\}$ (see (43)) is increasing. Note that $\left\{D_{\varphi}^{f}\left(x_{n}, u\right)\right\}$ is bounded by (76). It follows that $\lim _{n \rightarrow+\infty} D_{\varphi}^{f}\left(x_{n}, u\right)$ exists. By (78), we obtain

$$
\lim _{n \rightarrow+\infty} D_{\varphi}^{f}\left(x_{n+1}, x_{n}\right)=0 .
$$

One can see that $\lim _{n \rightarrow+\infty} f\left(x_{n}\right)=0$. Using Proposition 10, we obtain that $\lim _{n \rightarrow+\infty}\left\|x_{n}-x_{n+1}\right\|=0$. Since $x_{n+1} \in C_{n+1}$, we have

$$
\begin{aligned}
0 \leq & D_{\varphi}\left(x_{n+1}, y_{n}\right)+D_{\varphi}\left(y_{n}, x_{n}\right) \\
\leq & D_{\varphi}\left(x_{n+1}, y_{n}\right)+D_{\varphi}\left(y_{n}, x_{n}\right) \\
& +\left\langle y_{n}-x_{n+1}, J_{\varphi}\left(x_{n}\right)-J_{\varphi}\left(y_{n}\right)\right\rangle \\
& =D_{\varphi}\left(x_{n+1}, x_{n}\right) .
\end{aligned}
$$

Using (79), we obtain that

$$
\lim _{n \rightarrow+\infty}\left[D_{\varphi}\left(x_{n+1}, y_{n}\right)+D_{\varphi}\left(y_{n}, x_{n}\right)\right]=0,
$$

and hence $\lim _{n \rightarrow+\infty} D_{\varphi}\left(y_{n}, x_{n}\right)=0$. Note that $\left\{x_{n}\right\}$ is bounded. Then, one can see from Proposition 10 that $\left\{y_{n}\right\}$ is bounded and

$$
\lim _{n \rightarrow+\infty}\left\|x_{n}-y_{n}\right\|=0
$$

This implies that

$$
\lim _{n \rightarrow+\infty}\left\|x_{n}-T_{n} x_{n}\right\|=0 .
$$

Since the family $\mathscr{T}:=\left\{T_{n}: n \in \mathbb{N}\right\}$ has property $(\mathscr{A})$ with respect to $T$, it follows from (83) that $\lim _{n \rightarrow+\infty}\left\|x_{n}-T x_{n}\right\|=$ 0 .

Step 4 . The sequence $\left\{x_{n}\right\}$ converges strongly to $\Pi_{F(T)}^{\varphi, f}(u)$.

Since $\left\{x_{n}\right\}$ is bounded, there exists a subsequence $\left\{x_{n_{i}}\right\}$ of $\left\{x_{n}\right\}$ such that $x_{n_{i}} \rightarrow z \in C$. Hence $z \in \widehat{F}(T)=F(T)$. From Proposition 19, $F(T)$ is closed and convex. The nonemptiness of $F(T)$ implies that the generalized projection $\Pi_{F(T)}^{\varphi, f}$ is well defined. Note that $x_{n}=\Pi_{C_{n}}^{\varphi, f}(u)$ and $F(T)$ is contained in $C_{n}$; we have

$$
D_{\varphi}^{f}\left(x_{n}, u\right) \leq D_{\varphi}^{f}\left(\Pi_{F(T)}^{\varphi, f}(u), u\right)
$$

Therefore, we conclude from Proposition 16 that $\left\{x_{n}\right\}$ converges strongly to $\Pi_{F(T)}^{\varphi, f}(u)$.

Theorem 29. Let $X$ be a uniformly convex Banach space with a uniformly Gâteaux differentiable norm. Let $\varphi$ be a gauge function, $C$ a nonempty closed convex subset of $X$, and $f: X \rightarrow[0,+\infty]$ a proper, convex, lower semicontinuous function with $C \subset \operatorname{dom}(f)$. Let $A: X \rightarrow 2^{X^{*}}$ be a monotone operator with $A^{-1} 0 \neq \emptyset$ satisfying the following condition:

$$
D(A) \subset C \subset \bigcap_{r>0} J_{\varphi}^{-1} \mathscr{R}\left(J_{\varphi}+r A\right) .
$$

Let $\lambda$ be a positive real number; for $u$ in $C$ and $C_{1}=C$ with $x_{1}=\Pi_{C_{1}}^{\varphi, f}(u)$, define a sequence $\left\{x_{n}\right\}$ in $C$ as follows:

$$
\begin{gathered}
y_{n}=\left(J_{\varphi}+\lambda A\right)^{-1} J_{\varphi} x_{n}, \\
C_{n+1}=\left\{z \in C_{n}:\left\langle z-y_{n}, J_{\varphi}\left(x_{n}\right)-J_{\varphi}\left(y_{n}\right)\right\rangle \leq 0\right\}, \\
x_{n+1}=\Pi_{C_{n+1}}^{\varphi, f}(u), \quad \forall n \in \mathbb{N} .
\end{gathered}
$$

Then $\left\{x_{n}\right\}$ converges strongly to $\Pi_{A^{-1} 0}^{\varphi, f}(u)$.

Proof. Set $T:=\left(J_{\varphi}+\lambda A\right)^{-1} J_{\varphi}$. Note that $T$ is $\varphi$-firmly nonexpansive mapping from $C$ into $C$ and $F(T)=A^{-1} 0$. Further, every singleton family $\{T\}$ enjoys property $(\mathscr{A})$. Therefore, Theorem 29 follows from Theorem 28 .

Remark 30. Compared with other convergence theorems concerning proximal point algorithms in the literature (see, e.g., Agarwal et al. [35, Theorem 3.1]; Kamimura and Takahashi [26, Theorem 8]; Matsushita and Takahashi [32, Theorem 4.3]), Theorem 29 establishes a new proximal point algorithm for the problem of finding zeros of (not necessarily maximal) monotone operators in a uniformly convex Banach space with a uniformly Gâteaux differentiable norm.

We now derive an interesting new result.

Corollary 31. Let $C$ be a nonempty closed convex subset of real Hilbert space $H$, and let $f: X \rightarrow[0,+\infty]$ be a proper, convex, lower semicontinuous function with $C \subset \operatorname{dom}(f)$. Let $A \subset H \times H$ be a monotone operator satisfying condition (71) such that $A^{-1} 0 \neq \emptyset$. Let $\left\{\lambda_{n}\right\}$ be a sequence in $(0, \infty)$ such that $\lim _{n \rightarrow+\infty} \lambda_{n}=+\infty$, for $u$ in $C$, let $C_{1}=C$ with $x_{1}=P_{C_{1}}^{f}(u)$, and define a sequence $\left\{x_{n}\right\}$ in $C$ as follows:

$$
\begin{gathered}
y_{n}=\left(I+\lambda_{n} A\right)^{-1} x_{n}, \\
C_{n+1}=\left\{z \in C_{n}:\left\langle z-y_{n}, x_{n}-y_{n}\right\rangle \leq 0\right\}, \\
x_{n+1}=P_{C_{n+1}}^{f}(u), \quad \forall n \in \mathbb{N} .
\end{gathered}
$$

Then $\left\{x_{n}\right\}$ converges strongly to $P_{A^{-1} 0}^{f}(u)$.

Proof. Set $T:=R_{\lambda}^{A}=(I+\lambda A)^{-1}$ for $\lambda>0$ and $T_{n}:=(I+$ $\left.\lambda_{n} A\right)^{-1}$ for all $n$ in $\mathbb{N}$. Note that $T$ is a firmly nonexpansive mapping from $C$ into $C$ and $F(T)=A^{-1} 0$. From (83), we have $\lim _{n \rightarrow+\infty}\left\|x_{n}-T_{n} x_{n}\right\|=0$. Example 27 implies that $\mathscr{T}:=$ $\left\{T_{r}: r>0\right\}$ has property $(\mathscr{A})$. It follows that $\lim _{n \rightarrow+\infty} \| x_{n}-$ $T x_{n} \|=0$. Therefore, Corollary 31 follows from Theorem 28.

Let $C$ be a nonempty, closed, and convex subset of a Banach space $X$. Let $\Theta: C \times C \rightarrow \mathbb{R}$ be a bi-function, $A: C \rightarrow X^{*}$ a nonlinear operator, and $\psi: C \rightarrow \mathbb{R}$ 
a real-valued function. We assume the following conditions are all satisfied.

(A1) $\Theta(x, x)=0$ for all $x$ in $C$.

(A2) $\Theta$ is monotone; that is, $\Theta(x, y)+\Theta(y, x) \leq 0$ for all $x, y$ in $C$.

(A3) for all $x, y, z$ in $C, \lim \sup _{t \downarrow 0} \Theta(t z+(1-t) x, y) \leq$ $\Theta(x, y)$.

(A4) for all $x$ in $C, \Theta(x, \cdot)$ is convex and lower semicontinuous.

Blum and Oettli [36] studied the following equilibrium problem (EP).

Find $\widehat{x}$ in $C$ such that

$$
\Theta(\widehat{x}, y) \geq 0, \quad \forall y \in C .
$$

The solution set of $(88)$ is denoted by $\operatorname{EP}(\Theta)$.

Following [37], we have the following lemma.

Lemma 32. Let $C$ be a nonempty closed convex subset of a reflexive, strictly convex, and smooth Banach space $X$, and let $\varphi$ be a gauge function. Let $\Theta: C \times C \rightarrow \mathbb{R}$ be a bi-function satisfying conditions (A1)-(A4), and let $r>0$ and $x \in X$. Then there exists $z$ in $C$ such that

$$
\Theta(z, y)+\frac{1}{r}\left\langle y-z, J_{\varphi} z-J_{\varphi} x\right\rangle \geq 0, \quad \forall y \in C .
$$

We now consider the following generalized mixed equilibrium problem (GMEP): find $z$ in $C$ such that

$$
\Theta(z, y)+\langle A z, y-z\rangle+\psi(y)-\psi(z) \geq 0, \quad \forall y \in C .
$$

The solution set of (90) is denoted by $\operatorname{GMEP}(\Theta, A, \psi)$. The following auxiliary generalized mixed equilibrium problem is an important tool for finding the solution of GMEP (90).

Let $r>0$. For a given point $x$ in $C$, find $z$ in $C$ such that

$$
\begin{aligned}
& \Theta(z, y)+\langle A z, y-z\rangle+\psi(y)-\psi(z)+\frac{1}{r}\left\langle J_{\varphi} z-J_{\varphi} x, y-z\right\rangle \\
& \geq 0, \quad \forall y \in C .
\end{aligned}
$$

The existence of a solution of the auxiliary mixed equilibrium problem (91) is guaranteed by [37].

Let $C$ be a nonempty closed convex subset of a reflexive, strictly convex, and smooth Banach space $X$ and let $\varphi$ be a gauge function. Let $A: C \rightarrow X^{*}$ be continuous and monotone, $\Theta: C \times C \rightarrow \mathbb{R}$ a bi-function satisfying conditions (A1)-(A4), and $\psi: C \rightarrow \mathbb{R}$ a lower semicontinuous and convex function. For $r>0$, define the mapping $T_{r}^{(\Theta, A, \psi)}$ : $X \rightarrow 2^{C}$ as follows:

$$
\begin{aligned}
T_{r}^{(\Theta, A, \psi)}(x) & \\
= & \{z \in C: \Theta(z, y)+\langle A z, y-z\rangle+\psi(y) \\
& \left.\quad-\psi(z)+\frac{1}{r}\left\langle J_{\varphi} z-J_{\varphi} x, y-z\right\rangle \geq 0, \forall y \in C\right\} .
\end{aligned}
$$

Lemma 33 (see [37]). One has the following.

(1) $T_{r}^{(\Theta, A, \psi)}$ is single-valued.

(2) $T_{r}^{(\Theta, A, \psi)}$ is a $\varphi$-firmly nonexpansive mapping; that is, for all $x, y$ in $X$,

$$
\begin{gathered}
\left\langle T_{r}^{(\Theta, A, \psi)} x-T_{r}^{(\Theta, A, \psi)} y, J_{\varphi} T_{r}^{(\Theta, A, \psi)} x-J_{\varphi} T_{r}^{(\Theta, A, \psi)} y\right\rangle \\
\leq\left\langle T_{r}^{(\Theta, A, \psi)} x-T_{r}^{(\Theta, A, \psi)} y, J_{\varphi} x-J_{\varphi} y\right\rangle .
\end{gathered}
$$

(4) $F\left(T_{r}^{(\Theta, A, \psi)}\right)=G M E P(\Theta, A, \psi)$.

(5) $\operatorname{GMEP}(\Theta, A, \psi)$ is closed and convex.

(6) For all $x$ in $F\left(T_{r}^{(\Theta, A, \psi)}\right)$ and $y$ in $X$, one has

$D_{\varphi}\left(x, T_{r}^{(\Theta, A, \psi)} y\right)+D_{\varphi}\left(T_{r}^{(\Theta, A, \psi)} y, y\right) \leq D_{\varphi}(x, y)$.

The following theorem establishes the strong convergence of the proximal-projection method for solving generalized mixed equilibrium problems in the framework of uniformly convex Banach spaces.

Theorem 34. Let $C$ be a nonempty closed convex subset of a uniformly convex Banach space $X$ with a uniformly Gâteaux differentiable norm. Let $\varphi$ be a gauge function and let $f: X \rightarrow$ $[0, \infty]$ be a proper, convex, lower semicontinuous function with $C \subset \operatorname{dom}(f)$. Let $A: C \rightarrow X^{*}$ be continuous and monotone, $\Theta: C \times C \rightarrow \mathbb{R}$ a bi-function satisfying conditions (A1)$(A 4)$, and $\psi: C \rightarrow \mathbb{R}$ a lower semicontinuous and convex function. Assume that $\operatorname{GMEP}(\Theta, A, \psi, \varphi) \neq \emptyset$. For $u$ in $C, r>$ 0 , and $C_{1}=C$ with $x_{1}=\Pi_{C_{1}}^{\varphi, f}(u)$, define a sequence $\left\{x_{n}\right\}$ in $C$ as follows:

$$
\begin{gathered}
y_{n}=T_{r}^{(\Theta, A, \psi)} x_{n}, \\
C_{n+1}=\left\{z \in C_{n}:\left\langle z-y_{n}, J_{\varphi}\left(x_{n}\right)-J_{\varphi}\left(y_{n}\right)\right\rangle \leq 0\right\}, \\
x_{n+1}=\Pi_{C_{n+1}}^{\varphi, f}(u) \quad \forall n \in \mathbb{N} .
\end{gathered}
$$

Then $\left\{x_{n}\right\}$ strongly converges to $\prod_{F\left(T_{r}^{(\Theta, A, \psi)}\right)}^{\varphi, f}(u)$.

Proof. Note that $T_{r}^{(\Theta, A, \psi)}$ is a $\varphi$-firmly nonexpansive mapping from $C$ into $C$ and $F\left(T_{r}^{(\Theta, A, \psi)}\right)=\operatorname{GMEP}(\Theta, A, \psi, \varphi)$. Therefore, Theorem 34 follows from Theorem 28 .

\section{Acknowledgments}

This paper was initiated while D. R. Sahu was visiting the National Sun Yat-Sen University, Kaohsiung, Taiwan as a visiting professor. He would like to thank the Department of Applied Mathematics there for the warm hospitality. Professor Ngai-Ching Wong gave the encouragement and useful comments which were very important for this research. Both authors are also very grateful to the referees for their careful reading and many helpful suggestions. This research is partially supported by Taiwan NSC Grant 99-2115-M-110007-MY3 and the National Sun Yat-Sen University. 


\section{References}

[1] H. H. Bauschke, J. M. Borwein, and P. L. Combettes, "Bregman monotone optimization algorithms," SIAM Journal on Control and Optimization, vol. 42, no. 2, pp. 596-636, 2003.

[2] T. Bonesky, K. S. Kazimierski, P. Maass, F. Schöpfer, and T. Schuster, "Minimization of Tikhonov functionals in Banach spaces," Abstract and Applied Analysis, vol. 2008, Article ID 192679, 19 pages, 2008.

[3] L. M. Brègman, "A relaxation method of finding a common point of convex sets and its application to the solution of problems in convex programming," USSR Computational Mathematics and Mathematical Physics, vol. 7, pp. 200-217, 1967.

[4] D. Butnariu and A. N. Iusem, Totally Convex Functions for Fixed Points Computation and Infinite Dimensional Optimization, vol. 40, Kluwer Academic Publishers, Dordrecht, The Netherlands, 2000.

[5] Y. Censor and A. Lent, "An iterative row-action method for interval convex programming," Journal of Optimization Theory and Applications, vol. 34, no. 3, pp. 321-353, 1981.

[6] Y. I. Alber, "Generalized projection operators in Banach spaces: properties and applications," in Proceedings of the Israel Seminar, vol. 1 of Functional Differential Equation, pp. 1-21, Ariel, Israel, 1993.

[7] Y. I. Alber, "Metric and generalized projection operators in Banach spaces: properties and applications," in Theory and Applications of Nonlinear Operators of Accretive and Monotone Type, vol. 178, pp. 15-50, Dekker, New York, NY, USA, 1996.

[8] Y. Alber and S. Guerre-Delabriere, "On the projection methods for fixed point problems," Analysis, vol. 21, no. 1, pp. 17-39, 2001.

[9] Y. I. Alber and A. I. Notik, "On some estimates for projection operators in Banach spaces," Communications on Applied Nonlinear Analysis, vol. 2, no. 1, pp. 47-55, 1995.

[10] J. Li, “The generalized projection operator on reflexive Banach spaces and its applications," Journal of Mathematical Analysis and Applications, vol. 306, no. 1, pp. 55-71, 2005.

[11] W. Song and Z. Cao, "The generalized decomposition theorem in Banach spaces and its applications," Journal of Approximation Theory, vol. 129, no. 2, pp. 167-181, 2004.

[12] Y. Su, H.-K. Xu, and X. Zhang, "Strong convergence theorems for two countable families of weak relatively nonexpansive mappings and applications," Nonlinear Analysis. Theory, Methods \& Applications, vol. 73, no. 12, pp. 3890-3906, 2010.

[13] R. P. Agarwal, D. O'Regan, and D. R. Sahu, Fixed Point Theory for Lipschitzian-Type Mappings with Applications, vol. 6 of Topological Fixed Point Theory and Its Applications, Springer, New York, NY, USA, 2009.

[14] I. Cioranescu, Geometry of Banach Spaces, Duality Mappings and Nonlinear Problems, vol. 62, Kluwer Academic Publishers Group, Dordrecht, The Netherlands, 1990.

[15] T.-C. Lim, H. K. Xu, and Z. B. Xu, "Some $L^{p}$ inequalities and their applications to fixed point theory and approximation theory," in Progress in Approximation Theory, pp. 609-624, Academic Press, New York, NY, USA, 1991.

[16] C. Zălinescu, Convex Analysis in General Vector Spaces, World Scientific Publishing, River Edge, NJ, USA, 2002.

[17] J.-P. Penot and R. Ratsimahalo, "On the Yosida approximation of operators," Proceedings of the Royal Society of Edinburgh Section A, vol. 131, no. 4, pp. 945-966, 2001.

[18] H. H. Bauschke, J. M. Borwein, and P. L. Combettes, "Essential smoothness, essential strict convexity, and Legendre functions in Banach spaces," Communications in Contemporary Mathematics, vol. 3, no. 4, pp. 615-647, 2001.

[19] D. Butnariu, A. N. Iusem, and E. Resmerita, "Total convexity for powers of the norm in uniformly convex Banach spaces," Journal of Convex Analysis, vol. 7, no. 2, pp. 319-334, 2000.

[20] S. Reich and S. Sabach, "Two strong convergence theorems for a proximal method in reflexive Banach spaces," Numerical Functional Analysis and Optimization, vol. 31, no. 1-3, pp. 2244, 2010.

[21] D. Butnariu and G. Kassay, "A proximal-projection method for finding zeros of set-valued operators," SIAM Journal on Control and Optimization, vol. 47, no. 4, pp. 2096-2136, 2008.

[22] F. Schöpfer, T. Schuster, and A. K. Louis, "Metric and Bregman projections onto affine subspaces and their computation via sequential subspace optimization methods," Journal of Inverse and Ill-Posed Problems, vol. 16, no. 5, pp. 479-506, 2008.

[23] F. Schöpfer, T. Schuster, and A. K. Louis, "An iterative regularization method for the solution of the split feasibility problem in Banach spaces," Inverse Problems, vol. 24, no. 5, p. 20, 2008.

[24] F. Schöpfer and T. Schuster, "Fast regularizing sequential subspace optimization in Banach spaces," Inverse Problems, vol. 25, no. 1, p. 22, 2009.

[25] J.-J. Moreau, "Fonctions convexes duales et points proximaux dans un espace hilbertien," Comptes Rendus de l'Académie des Sciences, vol. 255, pp. 2897-2899, 1962.

[26] S. Kamimura and W. Takahashi, "Strong convergence of a proximal-type algorithm in a Banach space," SIAM Journal on Optimization, vol. 13, no. 3, pp. 938-945, 2002.

[27] D. Butnariu and E. Resmerita, "Bregman distances, totally convex functions, and a method for solving operator equations in Banach spaces," Abstract and Applied Analysis, vol. 2006, Article ID 84919, 39 pages, 2006.

[28] F. Kohsaka and W. Takahashi, "Existence and approximation of fixed points of firmly nonexpansive-type mappings in Banach spaces," SIAM Journal on Optimization, vol. 19, no. 2, pp. 824$835,2008$.

[29] K. Goebel and W. A. Kirk, Topics in Metric Fixed Point Theory, vol. 28, Cambridge University Press, Cambridge, UK, 1990.

[30] S. Reich and S. Sabach, "Existence and approximation of fixed points of Bregman firmly nonexpansive mappings in reflexive Banach spaces," in Fixed-Point Algorithms for Inverse Problems in Science and Engineering, vol. 49, pp. 301-316, Springer, New York, NY, USA, 2011.

[31] S. Reich, "A weak convergence theorem for the alternating method with Bregman distances," in Theory and Applications of Nonlinear Operators of Accretive and Monotone Type, vol. 178 of Lecture Notes in Pure and Applied Mathematics, pp. 313-318, Dekker, New York, NY, USA, 1996.

[32] S.-Y. Matsushita and W. Takahashi, "A strong convergence theorem for relatively nonexpansive mappings in a Banach space," Journal of Approximation Theory, vol. 134, no. 2, pp. 257266, 2005.

[33] G. Kassay, "The proximal points algorithm for reflexive Banach spaces," Universitatis Babeş-Bolyai. Studia. Mathematica, vol. 30, pp. 9-7, 1985.

[34] W. Takahashi, Nonlinear Functional Analysis, Fixed Point Theory and Its Applications, Yokohama Publishers, Yokohama, Japan, 2000.

[35] R. P. Agarwal, Y. J. Cho, and X. Qin, "Generalized projection algorithms for nonlinear operators," Numerical Functional Analysis and Optimization, vol. 28, no. 11-12, pp. 1197-1215, 2007. 
[36] E. Blum and W. Oettli, "From optimization and variational inequalities to equilibrium problems," The Mathematics Student, vol. 63, no. 1-4, pp. 123-145, 1994.

[37] S.-S. Zhang, "Generalized mixed equilibrium problem in Banach spaces," Applied Mathematics and Mechanics, vol. 30, no. 9, pp. 1105-1112, 2009. 


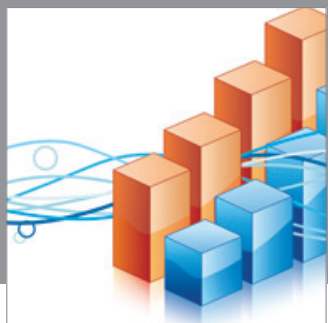

Advances in

Operations Research

mansans

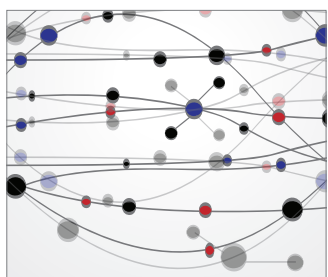

The Scientific World Journal
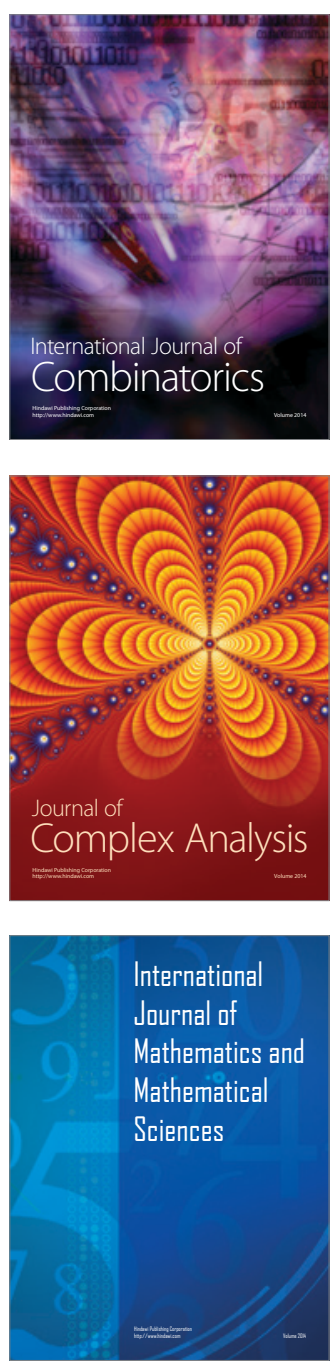
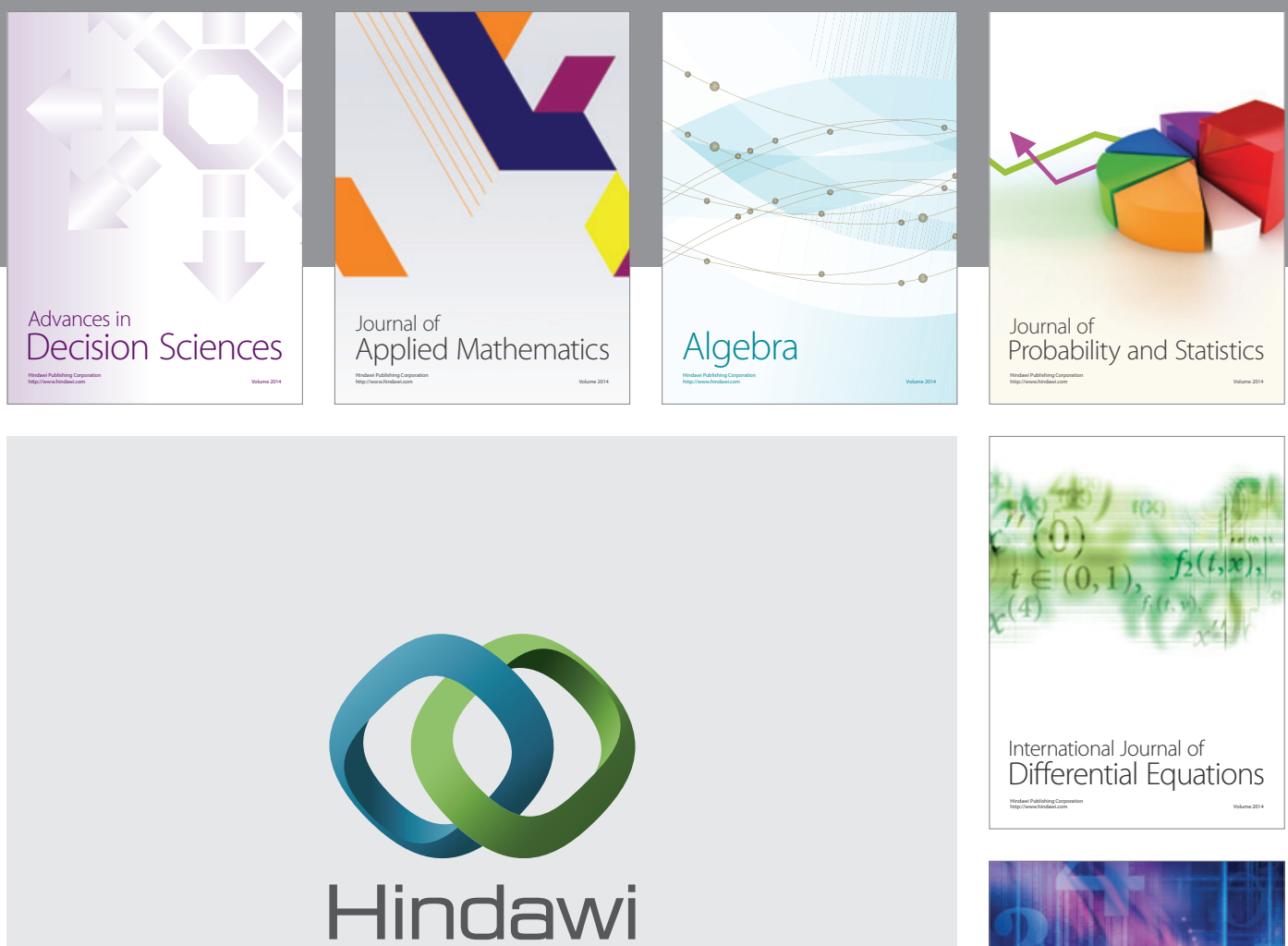

Submit your manuscripts at http://www.hindawi.com
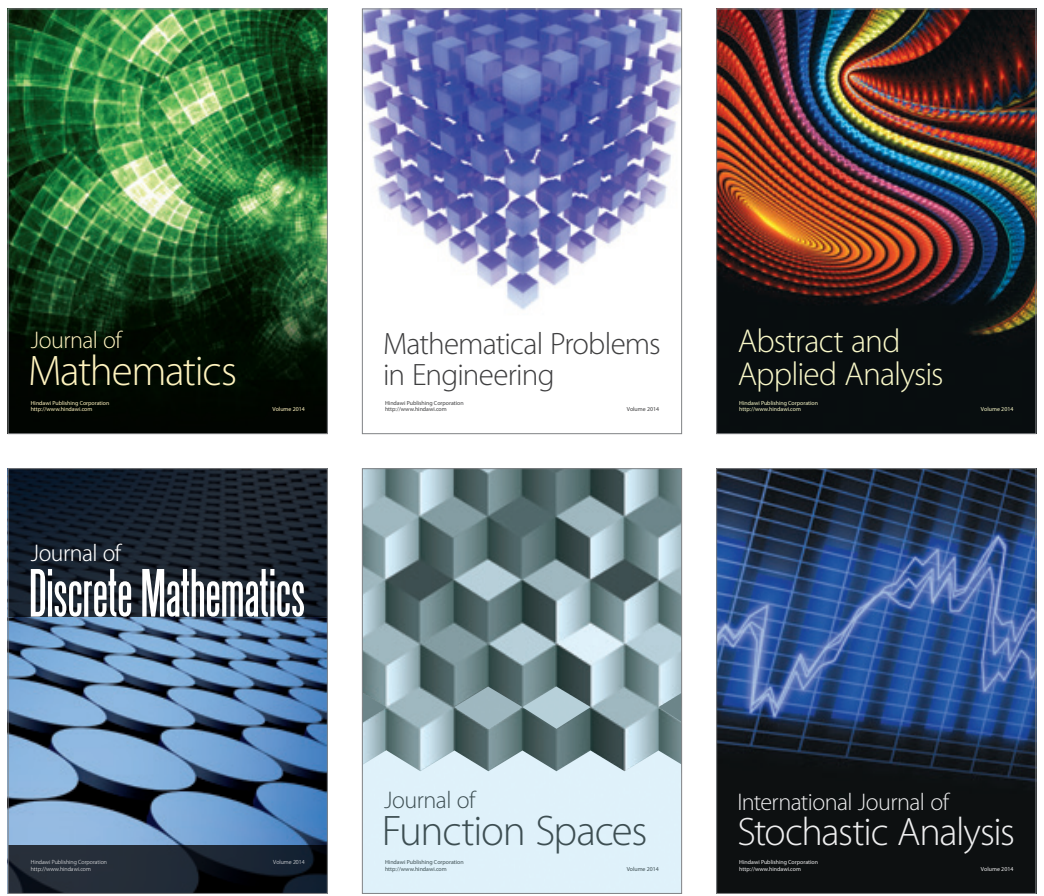

Journal of

Function Spaces

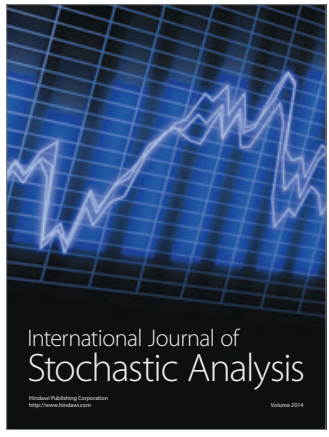

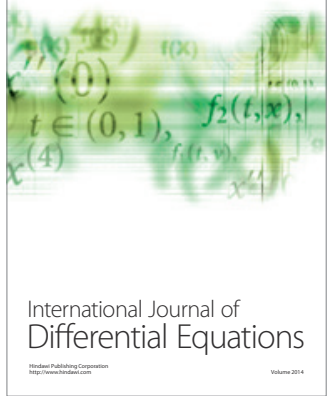
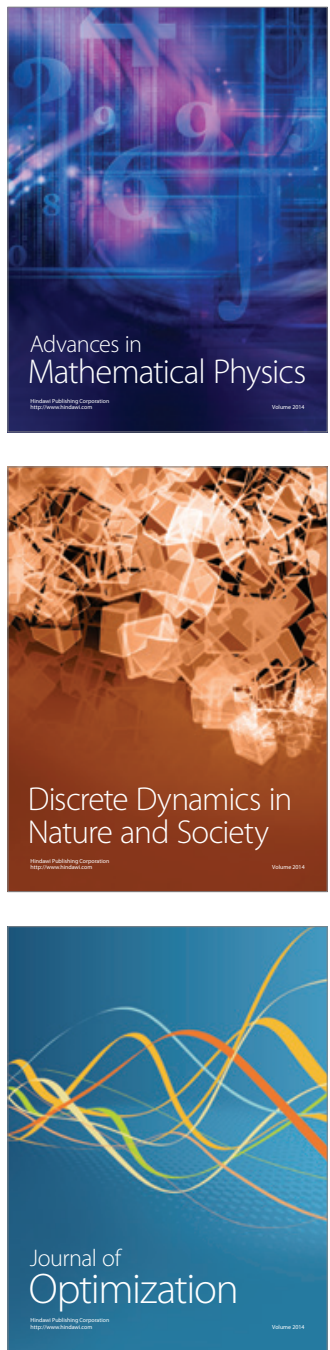